\title{
Erratum to: APC selectively mediates response to chemotherapeutic agents in breast cancer
}

\author{
Monica K. VanKlompenberg ${ }^{1,2}$, Claire O. Bedalov ${ }^{1,3}$, Katia Fernandez Soto ${ }^{1,3}$ and Jenifer R. Prosperi ${ }^{1,2,3^{*}}$
}

\section{Erratum}

After publication of the original article [1], it is noticed that all the Figures in the HTML version of the article are incorrect, please see the correct figures below. We apologize for any inconvenience caused.

\footnotetext{
Author details

${ }^{1}$ Harper Cancer Research Institute, A134 Harper Hall, 1234 Notre Dame Ave., South Bend, IN 46617, USA. Department of Biochemistry and Molecular Biology, Indiana University School of Medicine - South Bend, South Bend, IN, USA. ${ }^{3}$ Department of Biological Sciences, University of Notre Dame, Notre Dame, IN, USA.
}

Received: 13 November 2016 Accepted: 13 November 2016

Published online: 28 November 2016

\section{Reference}

1. VanKlompenberg MK, et al. APC selectively mediates response to

chemotherapeutic agents in breast cancer. BMC Cancer. 2015;15:457. doi:10. 1186/s12885-015-1456-x.

\footnotetext{
* Correspondence: jrprospe@iupui.edu

${ }^{1}$ Harper Cancer Research Institute, A134 Harper Hall, 1234 Notre Dame Ave. South Bend, IN 46617, USA

${ }^{2}$ Department of Biochemistry and Molecular Biology, Indiana University

School of Medicine - South Bend, South Bend, IN, USA
} 


$\begin{array}{lccc}\text { A } & & & \\ & A p c^{+/+} & A p c^{M i n} /+ & \text { Fold change } \\ \text { ABCG2/BCRP } & 25011 & 7359 & 0.29 \\ \text { MDR1 } & 85.45 & 276.6 & 3.24\end{array}$

B

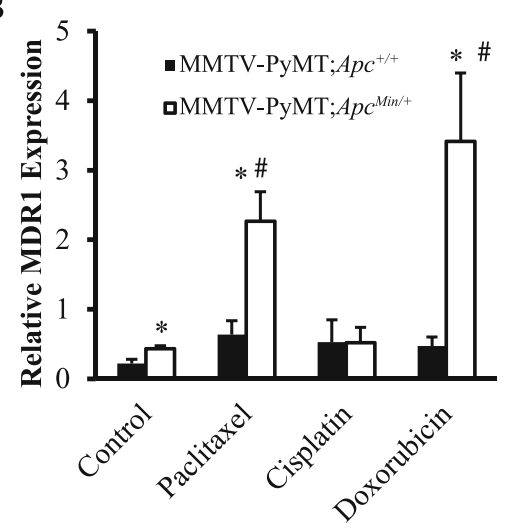

$\mathrm{C}$

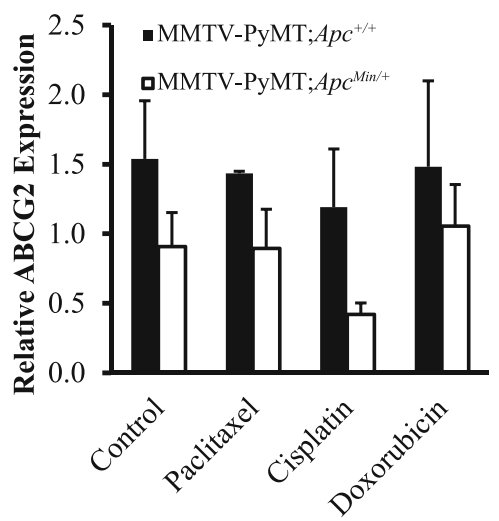

$\mathrm{D}$

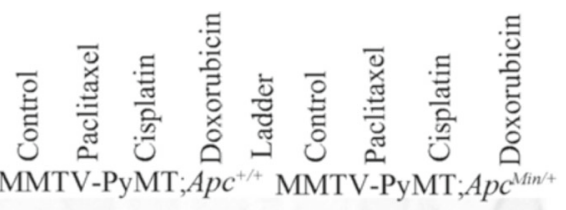

MDR1

$\mathrm{ABCG} 2-\cdots+$

Actin

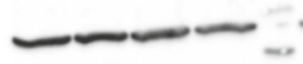

$\mathrm{E}$

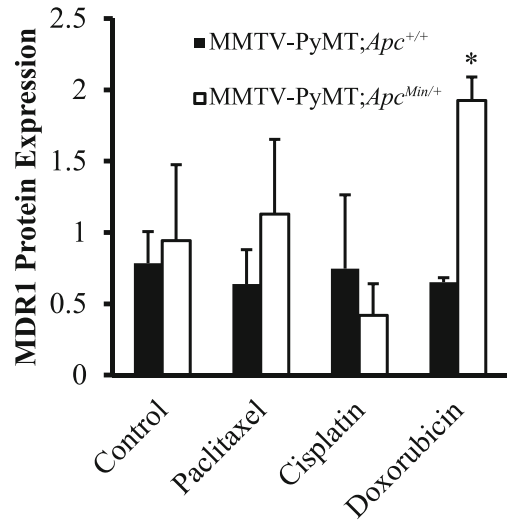

$\mathrm{F}$

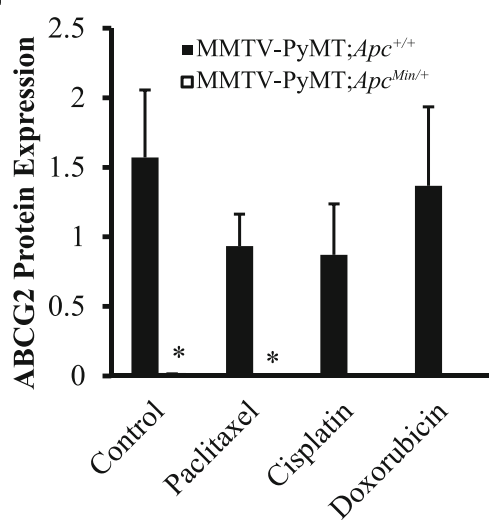

Fig. 1 Gene expression of ATP-dependent binding cassette transporters. a Microarray analysis of mammary glands from Apc ${ }^{\mathrm{Min} /+}$ and $A p c^{+/+}$mice at d16 of lactation show a decrease in ABCG2 and increase in MDR1 expression due to Apc mutation. b MDR1 gene expression in cells from MMTV-PyMT;ApC $C^{\text {Min/+ }}$ and MMTV-PyMT:ApC ${ }^{+/+}$mice after $24 \mathrm{~h}$ treatment with either solvent control, paclitaxel, cisplatin or doxorubicin. MDR1 expression was significantly increased in cells from MMTV-PyMT:ApC ${ }^{\mathrm{Min} /+}$ mice after treatment with paclitaxel and doxorubicin but not cisplatin. c ABCG2 gene expression in cells from MMTVPyMT:Apc ${ }^{\mathrm{Min} /+}$ and MMTV-PyMT;Apc ${ }^{+/+}$mice after treatment for $24 \mathrm{~h}$ with either solvent control, paclitaxel, cisplatin or doxorubicin. ABCG2 expression was not different between MMTV-PyMT;Apc ${ }^{\text {Min/+ }}$ and MMTV-PyMT:Apc ${ }^{+/+}$cells and chemotherapy treatment had no effect on ABCG2 expression. $\mathbf{d}$ Representative western blots for MDR1 and ABCG2 in cells from MMTV-PyMT:Apc ${ }^{\mathrm{Min} /+}$ and MMTV-PyMT;Apc $c^{+/+}$mice after treatment for $24 \mathrm{~h}$ with either solvent control, paclitaxel, cisplatin or doxorubicin. e Quantification of MDR1 western blots shows that MMTV-PyMT:Apc ${ }^{\text {Min/ }}$ cells have enhanced MDR1 expression when treated with doxorubicin. $\mathbf{f}$ Quantification of ABCG2 western blots shows that MMTV-PyMT:Apc ${ }^{+/+}$cells have elevated ABCG2 protein expression compared to MMTV-PyMT:Apc ${ }^{\text {Min/t }}$ cells. Results in $\mathbf{b}$, $\mathbf{c}$, e and $\mathbf{f}$ are shown as the means \pm SEM from 3 independent experiments; ${ }^{*} P<0.05$ when comparing MMTVPyMT:Apc ${ }^{\mathrm{Min} /+}$ to MMTV-PyMT:Apc ${ }^{+/+}$cells and \#P<0.05 when comparing MMTV-PyMT;ApC ${ }^{\text {Min/+ }}$ cells treated with solvent control or chemotherapy agent 
a

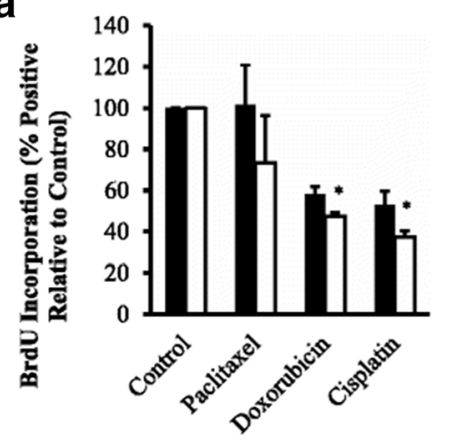

b

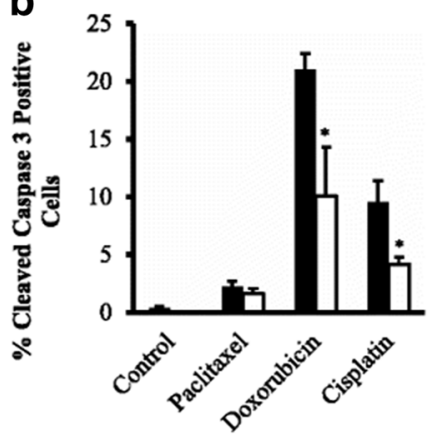

MMTV-PyMT;Apc $c^{+/ \alpha} \quad \square$ MMTV-PyMT;Apc $c^{\text {dinat+ }}$

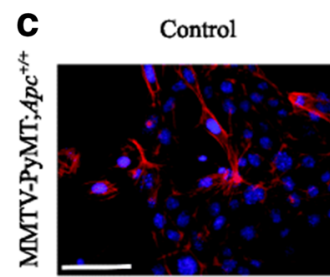

Paclitaxel

Doxorubicin

Cisplatin
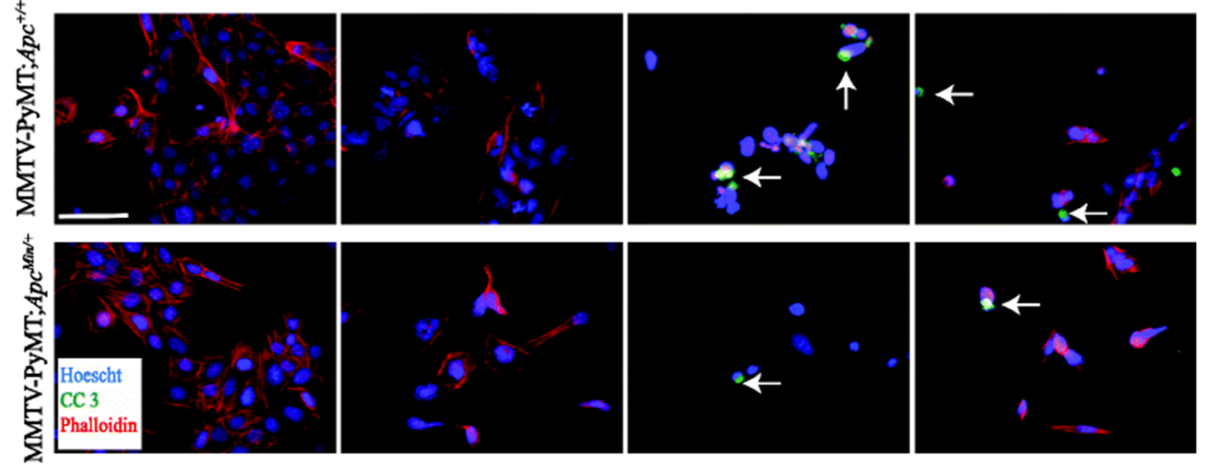

Fig. 2 Cell proliferation and apoptosis in MMTV-PyMT;Apc $C^{\text {Min/+ }}$ and MMTV-PyMT;Apc ${ }^{+/+}$cells after treatment with paclitaxel, cisplatin and doxorubicin. a Cell proliferation as measured by BrdU incorporation after chemotherapuetic treatment. MMTV-PyMT;Apc ${ }^{\text {Min/+ }}$ cells showed a modest decrease in proliferation after treatment with cisplatin and doxorubicin compared to MMTV-PyMT:Apc ${ }^{+/+}$cells. $\mathbf{b}$ Apoptosis as measured by cleaved caspase 3

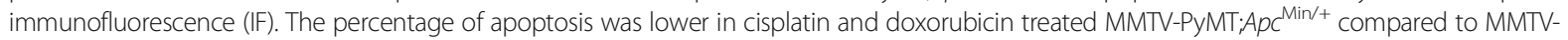
PyMT; $\mathrm{Apc}^{+/+}$cells while paclitaxel treatment did not affect apoptosis levels. c Representative images of cleaved caspase 3 (CC3) IF. The scale bar is equal to 200 microns. White arrows are representative cleaved caspase 3 positive cells. Data are shown as the means \pm SEM from 3 independent experiments; ${ }^{*} P<0.05$ 
a

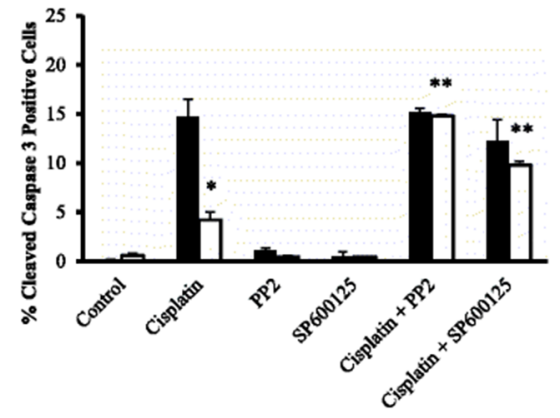

MMTV-PyMT;Apc $c^{+/+}$

\section{b}

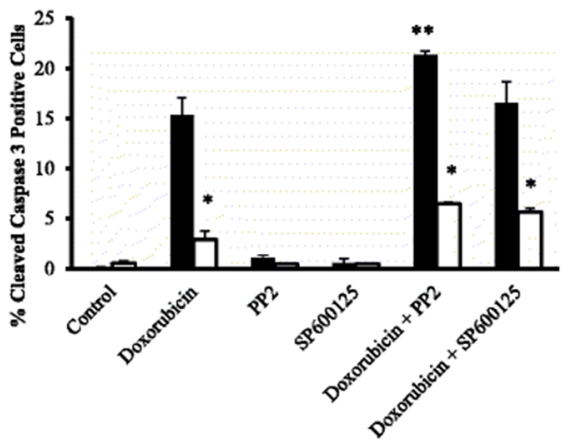

$\square$ MMTV-PуMT;Apc $c^{\text {Min/t+ }}$

Cisplatin + PP2

Cisplatin + SP600125

C

Control
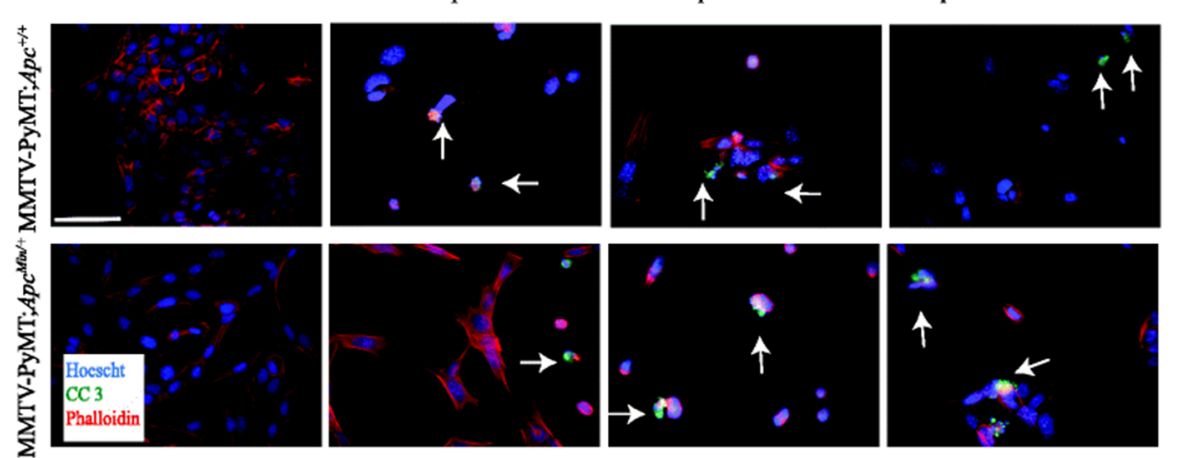
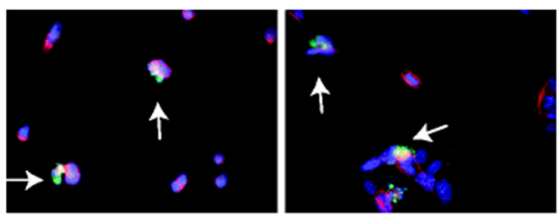

Fig. 3 Apoptosis in MMTV-PyMT;ApC ${ }^{\text {Min/+ }}$ and MMTV-PyMT;Apc ${ }^{+/+}$cells treated with chemotherapeutic drugs and targeted inhibitors. a Apoptosis was measured by cleaved caspase 3 IF in the presence of cisplatin. Treatment with cisplatin and either PP2 or SP600125 significantly increases apoptosis compared to cisplatin alone in MMTV-PyMT;Apc ${ }^{\text {Min/t }}$ cells. No effect was observed with the addition of PP2 or SP600125 in the MMTVPyMT; $A \mathrm{Ac}^{+/+}$cells. b Apoptosis was measured by cleaved caspase 3 IF with doxorubicin treatments. c Representative cleaved caspase 3 IF images of cells treated with cisplatin and the targeted inhibitor. The scale bar is equal to 200 microns and arrows are used to depict specific cleaved caspase 3 (CC 3) positive cells in each image. Data are shown as the means \pm SEM from 3 independent experiments; ${ }^{*} P<0.05$ when comparing MMTV-PyMT;ApC ${ }^{\mathrm{Min} /+}$ to MMTV-PyMT;Apc ${ }^{+/+}$cells and ${ }^{* *} P<0.05$ when comparing the combination treatment versus a single agent (cisplatin or doxorubicin) 

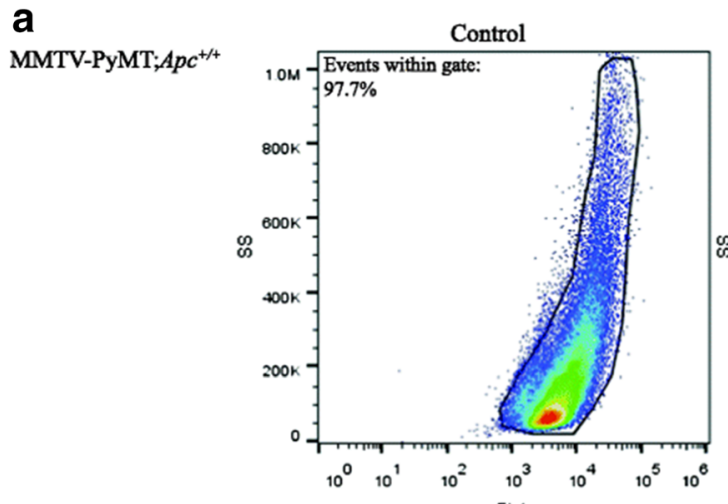

MMTV-PyMT; $A p c^{\text {Mint }}$
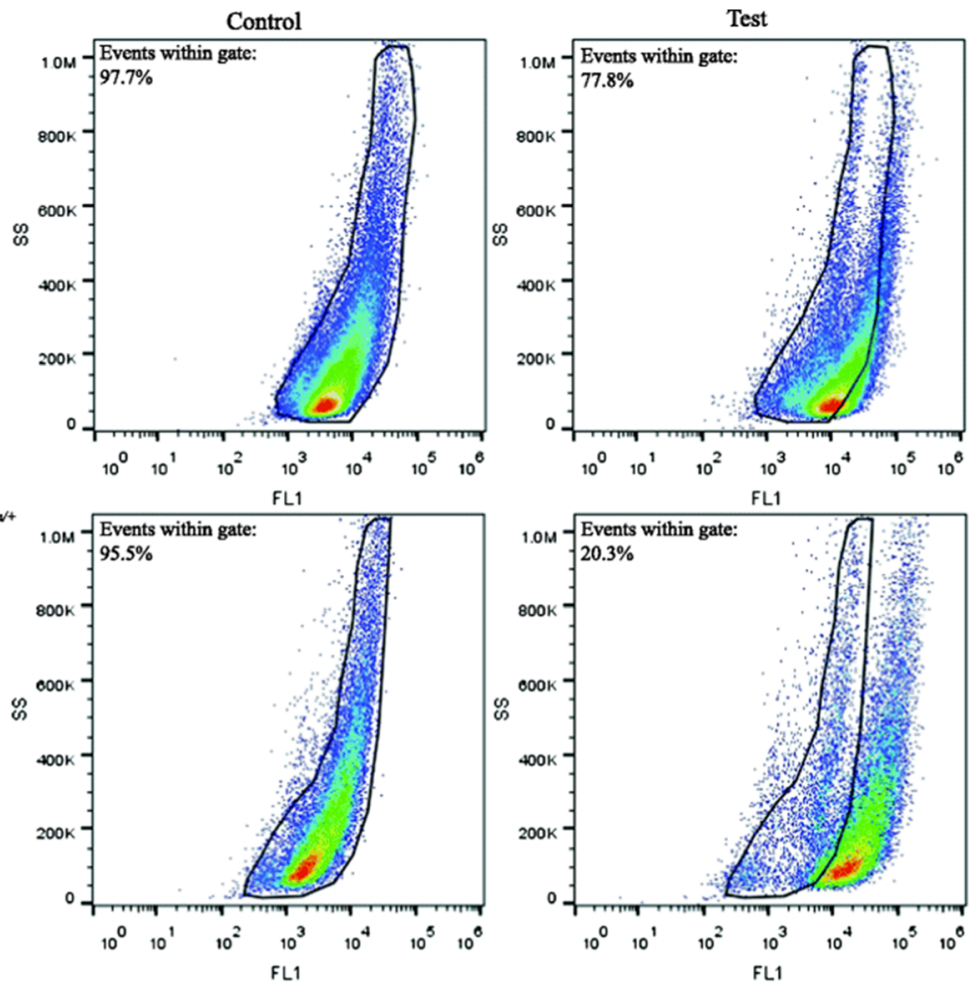

b

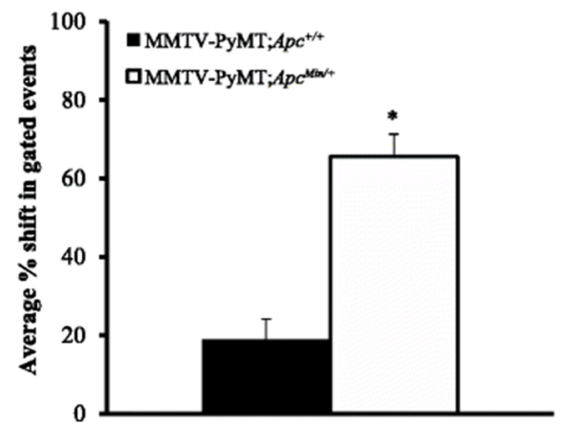

Fig. 4 MMTV-PyMT;Apc ${ }^{\mathrm{Min} /+}$ cells have higher aldehyde dehydrogenase (ALDH) enzyme activity than MMTV-PyMT;Apc ${ }^{+/+}$cells. ALDH activity was measured using an Aldefluor ${ }^{\mathrm{TM}}$ Kit. For each cell line a Control (+DEAB) and test (-DEAB) sample were run. a Representative FACS analysis of ALDH activity in MMTVPyMT; Apc ${ }^{+/+}$and MMTV-PyMT;Apc ${ }^{\mathrm{Min} /+}$ cells using the Aldefluorm assay. ALDH activity is increased in MMTV-PyMT;Apc ${ }^{\mathrm{Min} /+}$ cells. b The population of cells that shifted outside of the control population was calculated for each test sample, indicating ALDH activity. MMTVPyMT;Apc ${ }^{\text {Min/+ }}$ cells show a larger percentage of cells shifted outside of the control range than MMTV-PyMT;Apc ${ }^{+/+}$cells. Data are shown as the means \pm SEM from 3 independent experiments; ${ }^{*} P<0.05$ 

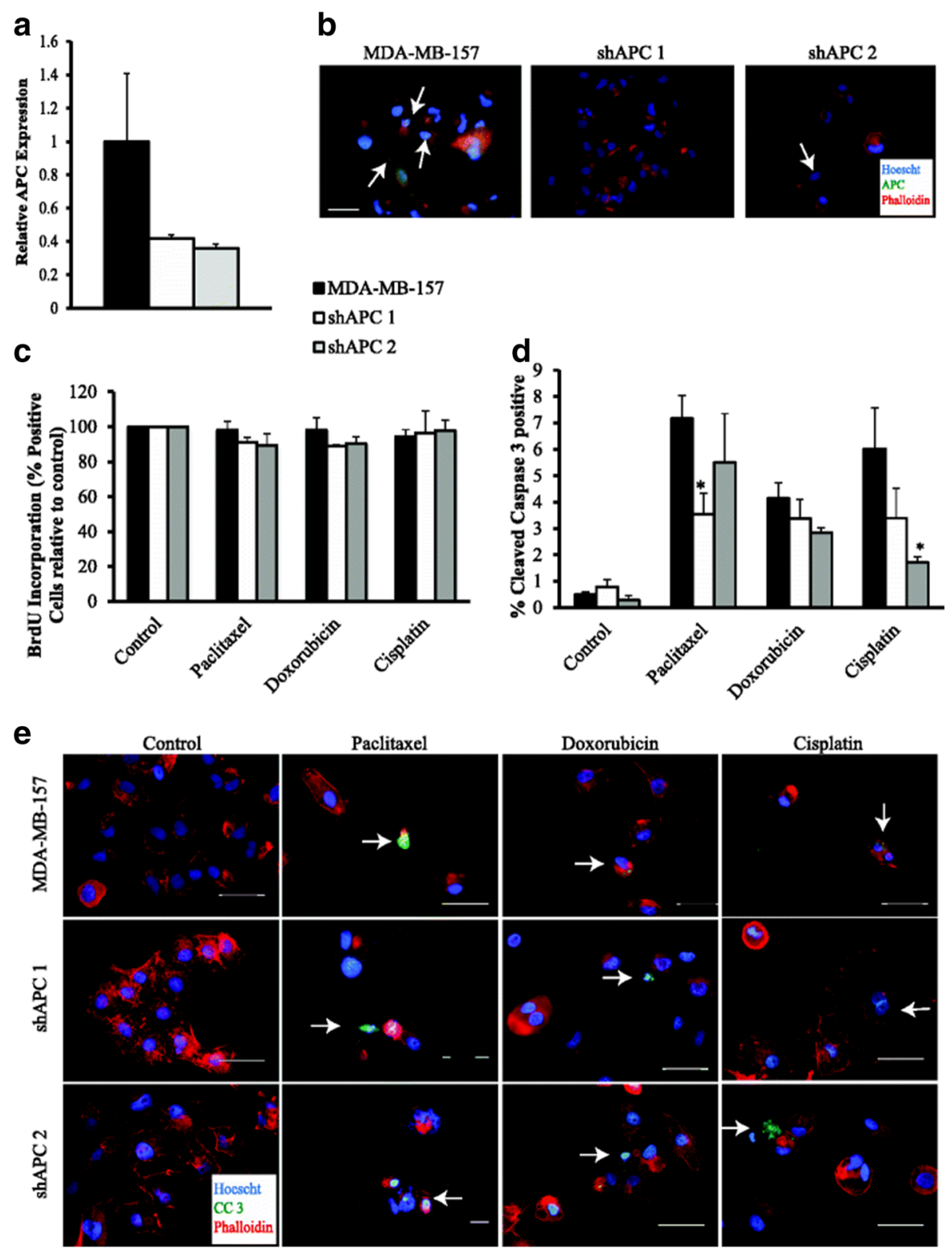

Fig. 5 APC knockdown in MDA-MB-157 cells impacts response to paclitaxel and cisplatin. a Quantitative RT-PCR in MDA-MB-157 cells and shAPC constructs shows decreased level of APC in cells infected with the shAPC constructs. $\mathbf{b}$ Representative APC immunofluorescence images showing that APC knockdown cells have less APC protein compared to the MDA-MB-157 parent line. c Cell proliferation as measured by BrdU incorporation did not differ between the three cell lines after treatment with cisplatin, doxorubicin or paclitaxel. $\mathbf{d}$ Apoptosis as measured by cleaved caspase 3 IF. The percentage of apoptosis was lower in paclitaxel treated shAPC 1 and cisplatin treated shAPC 2 cells compared to MDA-MB-157 control cells. Doxorubicin treatment had no effect on rates of apoptosis. e Representative images of CC3 IF. Although there are a similar number of positive cells in many of the images, there are fewer total cells in those images representing treatments with a higher percent of apoptosis. The scale bar is equal to 100 microns (e) and 20 microns (b). Data are shown as the means \pm SEM from 3 independent experiments; $* P<0.05$ 